\title{
Level crossing probabilities for multipath acoustic processes with bimodal spectra
}

\author{
Anastassios N. Perakis and Dimitrios V. Lyridis \\ Department of Naval Architecture and Marine Engineering, The University of Michigan, Ann Arbor, \\ Michigan 48109
}

(Received 19 October 1988; accepted for publication 26 December 1989)

\begin{abstract}
A general method of evaluating upcrossing probabilities for a class of random processes consisting of two narrow-band signals is presented. One of the two significant frequencies of the corresponding bimodal spectra is assumed to be dominant. The method approximates the maxima of these processes by the corresponding values of the envelope processes. It is also assumed that the discrete processes of the maxima are Markov. The results have several applications. Two prominent examples are detection problems of multipath partially saturated processes in underwater acoustics and the problem of the structural reliability of marine diesel engine shafting systems.
\end{abstract}

PACS numbers: $43.60 . \mathrm{Cq}, 43.60 . \mathrm{Gk}$, 43.30.Vh

\section{INTRODUCTION}

The ocean acoustic detection problem has been studied for the general case of partially saturated propagation of narrow-band ocean acoustic multipath processes ${ }^{1,2}$ with special cases being the fully saturated ${ }^{3}$ and the unsaturated ${ }^{4}$ propagation, respectively. The pressure for these narrow-band ocean acoustic multipath processes is given by

$$
p(t)=X(t) \cos \omega_{0} t+Y(t) \sin \omega_{0} t .
$$

Assuming that the pressure process $p(t)$ has a large number of independent paths, processes $X(t)$ and $Y(t)$ can be assumed to be Gaussian.

This process has also been employed in the formulation of the problem of structural reliability of marine diesel engine propulsion shafting systems. ${ }^{5-7}$ That analysis is limited to the most common case, where only a single resonance (critical) is excited in any operating condition. Recent work by one of our colleagues has revealed important cases where two separate critical frequencies appear at essentially the same engine operating condition. In that example, each critical contributed roughly half of the stress at the critical engine rpm.

To treat such cases, we need to evaluate the upcrossing probability of a random process of the form

$$
\sum_{i=1}^{n}\left[X_{i}(t) \cos \omega_{i} t+Y_{i}(t) \sin \omega_{i} t\right], \quad n=2,
$$

where $X_{i}(t)$ and $Y_{i}(t)$ are Gaussian, stationary processes with known means, autocorrelations, and cross correlations. This calculation will also be very important for the underwater acoustic detection problem, when the pressure $p(t)$ mentioned above is the sum of two narrow-band processes, centered at two distinct frequencies. The general case (arbitrary $n$ ) for both the acoustic underwater detection and the marine shafting system structural reliability problem is very difficult to evaluate, but the $n=2$ case will be adequate for most problems anticipated in practice. Finally, processes $X_{i}(t)$ and $Y_{i}(t)$ may be assumed to be independent. This assumption is valid, because even if they are not, we can always consider a transformation to a new coordinate system, where they are independent.

\section{SPECTRAL ANALYSIS}

Our process has the form

$$
Z(t)=W_{1}(t)+W_{2}(t),
$$

where

$$
\begin{aligned}
& W_{1}(t)=X_{1}(t) \cos \omega_{1} t+Y_{1}(t) \sin \omega_{1} t \\
& W_{2}(2)=X_{2}(t) \cos \omega_{2} t+Y_{2}(t) \sin \omega_{2} t
\end{aligned}
$$

Processes $W_{1}(t)$ and $W_{2}(t)$ can be assumed to be narrow band, as their entire energy is concentrated in the neighborhood of $\omega_{1}$ and $\omega_{2}$, respectively. Hence, $Z(t)$ is the sum of two narrow-band processes, whose spectrum has its energy concentrated at the two frequencies $\omega_{1}$ and $\omega_{2}$.

Our approach for the evaluation of the upcrossing probability of our process is based on the approximation of the local maxima of $Z(t)$ by the values of the envelope process at the times that these maxima occur. ${ }^{5}$ Then a discrete time, two-state process is defined, with two states defined by the requirement that the value of $Z(t)$ exceeds or does not exceed (respectively) the specified threshold $Z_{0}$, during a time interval of length $T$.

Assuming that the local maxima of $Z(t)$ are approximately equal to the value of the envelope at the time they occur, the upcrossing probability is equal to the probability that at least one of the values of the envelope at $t_{1}, t_{2}, \ldots$ exceeds the value of $Z_{0}$ during the time period $T$, where $Z_{0}, T$ are both known. This approach was followed also for the one-frequency problem by Nikolaidis et al. ${ }^{6}$ The key to the following formulation is choosing the appropriate envelope that is tangent to our process at the peaks, since we approximate the local maxima of $Z(t)$ by the corresponding values of the envelope. 


\section{ENVELOPE PROCESS}

The envelope process for $W_{1}(t)$ is ${ }^{5}$

$$
R_{1}(t)=\sqrt{X_{1}^{2}(t)+Y_{1}^{2}(t)} .
$$

Similarly, the envelope process for $W_{2}(t)$ is

$$
R_{2}(t)=\sqrt{X_{2}^{2}(t)+Y_{2}^{2}(t)} .
$$

Here, $R_{1}(t)$ is derived as follows: We define

$$
W_{1}(t)=X_{1}(t) \cos \omega_{1} t+Y_{1}(t) \sin \omega_{1} t .
$$

Dividing and multiplying by $\sqrt{X_{1}^{2}(t)+Y_{1}^{2}(t)}$, we get

$$
\begin{aligned}
W_{1}(t)= & \sqrt{X_{1}^{2}(t)+Y_{1}^{2}(t)}\left(\frac{X_{1}(t)}{\sqrt{X_{1}^{2}(t)+Y_{1}^{2}(t)}} \cos \omega_{1} t\right. \\
& \left.+\frac{Y_{1}(t)}{\sqrt{X_{1}^{2}(t)+Y_{1}^{2}(t)} \sin \omega_{1} t}\right) \\
= & \sqrt{X_{1}^{2}(t)+Y_{1}^{2}(t)} \cos \left[\omega_{1} t-\theta_{1}(t)\right] \\
= & R_{1}(t) \cdot \cos \left[\omega_{1} t-\theta_{1}(t)\right] .
\end{aligned}
$$

Hence

$$
R_{1}(t)=\sqrt{X_{1}^{2}(t)+Y_{1}^{2}(t)}
$$

and

$$
\theta_{1}(t)=\tan ^{-1}\left[Y_{1}(t) / X_{1}(t)\right]
$$

As $W_{1}(t)$ is a narrow-band process, $R_{1}(t)$ and $\theta_{1}(t)$ are slowly varying random processes. The same holds for $W_{2}(t)$.

For narrow-band random processes, several definitions of the envelope have been proposed. Among these, the envelope defined by Cramer and Leadbetter ${ }^{8}$ is mathematically convenient. For a Gaussian process $B(t)$, the Cramer-Leadbetter $(\mathrm{CL})$ envelope is defined as

$$
R_{B}(t)=\left[B^{2}(t)+\widehat{B}^{2}(t)\right]^{1 / 2},
$$

where $\widehat{B}(t)$ is the Hilbert transform of $B(t)$ (Toro and Cornell, Ref. 9), defined by

$$
\widehat{B}(t)=\int_{0}^{\infty}[\cos \omega t d V(u)-\sin \omega t d U(u)],
$$

and $U(u), V(u)$ are independent random processes with independent increments such that $B(t)$ can be expressed as

$$
B(t)=\int_{-\infty}^{+\infty}[\cos \omega t d U(u)+\sin \omega t d V(u)] .
$$

Now for bimodal processes such as $Z(t)$ with two major distant frequencies (Toro and Cornell, Ref. 9) the CL envelope is well defined and can be written as

$$
\begin{aligned}
R_{Z}(t)= & \left\{\left[W_{1}(t)+W_{2}(t)\right]^{2}\right. \\
& \left.+\left[\hat{W}_{1}(t)+\hat{W}_{2}(t)\right]^{2}\right\}^{1 / 2} .
\end{aligned}
$$

Some characteristics of the envelope are the following ${ }^{9}$ : The envelope should be much smoother than the process, free of periodic oscillations, and should follow the process closely, making contact near every peak and trough. However, all these properties cannot be satisfied by the CL envelope for $Z(t)$. In this case, there are two alternatives. Two envelopes can be defined in such a way that the first satisfies the smoothness and lack of periodicity conditions and the second coincides with the process itself near every peak. ${ }^{9}$ The first envelope that we mentioned is defined as the sum of the envelopes of $W_{1}(t), W_{2}(t)$ and is called "rectangular envelope." The second envelope is defined as $S(t)$ $=W_{1}(t)+R_{2}(t)$, where we make the assumption that $W_{1}(t)$ has the lower frequency of the two, $\omega_{1}$, and the higher one, $\omega_{2}$, is the corresponding frequency for $W_{2}(t)$. We will choose the second one, since our basic requirement for the envelope is to approximate the process near the peaks.

The "envelope" process chosen violates the nonperiodicity requirement, but this in our case is not important. What is important is that this "envelope" process is able to follow even the low-frequency peaks of $Z(t)$. On the other hand, the classical envelope that is found by demodulating at $\left(\omega_{1}+\omega_{2}\right) / 2$ is not well defined, because (as we shall see later) $\omega_{1} \ll \omega_{2}$, therefore the difference frequency is not "much less" than either carrier.

Finally, we are going to assume that we have one low frequency, $\omega_{1}$, and one high frequency, $\omega_{2}$, with $\omega_{1} \ll \omega_{2}$. The expression of the envelope process will be ${ }^{9}$ :

$$
\begin{aligned}
S(t)= & W_{1}(t)+R_{2}(t) \\
= & X_{1}(t) \cos \omega_{1} t+Y_{1}(t) \sin \omega_{1} t \\
& +\sqrt{X_{2}^{2}(t)+Y_{2}^{2}(t)} .
\end{aligned}
$$

In the following, the term "envelope process" will refer to the "envelope" process defined by (7).

\section{CALCULATION OF THE UPCROSSING PROBABILITY}

We will now consider a discrete-time random process with two states denoted by $U$ and $D$, defined by the requirements that the envelope of $Z(t)$ exceeds and does not exceed the threshold $Z_{0}$, respectively. The discrete process takes values at the times $0, t_{1}, t_{2}, \ldots$, where the maxima of the process $Z(t)$ occur. We can assume this discrete process as Markov, with one step dependence. ${ }^{10,11} \mathrm{We}$ want to calculate the probability of an upcrossing during a time interval $T$. This probability equals the probability that at least one of the maxima is greater than the threshold, $Z_{0}$, or

$$
\begin{aligned}
p \equiv & P\{\text { upcrossing in }[0, T]\} \\
= & P\left(\xi_{0}>Z_{0}\right)+P\left(\xi_{1}>Z_{0}, \xi_{0} \leqslant Z_{0}\right) \\
& +P\left(\xi_{2}>Z_{0}, \xi_{1} \leqslant Z_{0}, \xi_{2} \leqslant Z_{0}\right)+\cdots,
\end{aligned}
$$

where $\xi_{i}$ denotes the $i$ th maximum of the process.

Clearly, to evalute ( 8 ), we need to calculate the number $v$ of the maxima in the time interval $T$. Thus,

$P$ \{upcrossing in $[0, T]\}$

$$
\begin{aligned}
= & P(U)+P(D U)+P(D D U) \\
& +\cdots+P(D \cdots D U),
\end{aligned}
$$

where $D$ appears $\left(v^{-1}\right)$ times in the last term.

Let us now denote

$$
P(U)=c, \quad P(U / D)=b .
$$

It follows that

$$
P(D)=1-c, \quad P(D / D)=1-b .
$$

We assume that the process is Markov with one-step depen- 
dence. In other words, every maximum depends only on the previous one, ${ }^{12}$ or

$$
P(U / D)=P(U / D D)=\cdots=P(U / D D \cdots D) .
$$

For $P(U D)$, we have

$$
P(U D)=P(U / D) P(D)=b(1-c) .
$$

For $P(U D D)$, we have

$$
\begin{aligned}
P(U D D) & =P(U / D D) P(D D) \\
& =P(U / D D) P(D / D) P(D) \\
& =b(1-b)(1-c) .
\end{aligned}
$$

Similarly, we obtain

$$
P(U D D \cdots D)=b(1-b)^{v-2}(1-c) .
$$

Substituting the probabilities in Eqs. (10), (11), (12) into (8), we obtain

$P\{$ upcrossing in $[0, T]\}$

$$
\begin{aligned}
= & c+b(1-c)+b(1-b)(1-c) \\
& +b(1-b)^{2}(1-c)+\cdots \\
& +b(1-b)^{v-2}(1-c) \\
= & c+b(1-c)\left[1+(1-b)+(1-b)^{2}+\cdots\right. \\
& \left.+(1-b)^{v-2}\right] \\
= & 1-(1-c)(1-b)^{v-1} .
\end{aligned}
$$

Therefore, in order to calculate the upcrossing probability, we need to evaluate $b, c, v$.

The expressions for $b$ and $c$ are (see Nikolaidis and Perakis, Ref. 2)

$$
\begin{aligned}
c= & \int_{Z_{0}}^{\infty} f_{S}(s) d s, \\
b= & \left(\int_{-\infty}^{Z_{0}} \int_{Z_{0}}^{\infty} f_{S(t), S(t+\tau)}\left(s_{1}, s_{2}\right) d s_{2} d s_{1}\right) \\
& \times\left(\int_{-\infty}^{Z_{0}} f_{S}(s) d s\right)^{-1},
\end{aligned}
$$

where $f_{S}(s)$ and $f_{S(t), S(t+\tau)}\left(s_{1}, s_{2}\right)$ are the PDF and the joint PDF of the envelope process $S(t)$, respectively. We will next derive these two PDF's.

\section{CALCULATION OF THE FIRST ORDER PDF}

Our envelope process has the form

$$
\begin{aligned}
S(t)= & X_{1}(t) \cos \omega_{1} t+Y_{1}(t) \sin \omega_{1} t \\
& +\sqrt{X_{2}^{2}(t)+Y_{2}^{2}(t)} .
\end{aligned}
$$

Processes $X_{1}(t), Y_{1}(t), X_{2}(t)$, and $Y_{2}(t)$ are assumed at time $t$ jointly independent Gaussian random variables with known means and autocorrelations. Since $X_{1}(t)$ and $Y_{1}(t)$ are (independent) Gaussian random processes, every linear transformation $U(t)$ of $X_{1}(t)$ and $Y_{1}(t)$ is also a Gaussian random process, with mean:

$$
\mu_{U}=\mu_{X_{1}} \cos \omega_{1} t+\mu_{Y_{1}} \sin \omega_{1} t,
$$

where $\mu_{X_{1}}$ and $\mu_{Y_{1}}$ are the expected values of $X_{1}(t)$ and $Y_{1}(t)$, respectively, and second central moment:

$$
\sigma_{U}^{2}=\sigma_{X_{1}}^{2} \cos ^{2} \omega_{1} t+\sigma_{Y_{1}}^{2} \sin ^{2} \omega_{1} t,
$$

where $\sigma_{X_{1}}^{2}$ and $\sigma_{Y_{1}}^{2}$ are the second central moments of $X_{1}(t)$ and $Y_{1}(t)$, respectively. Consequently, the first-order PDF for random process

$$
\begin{gathered}
U(t)=X_{1}(t) \cos \omega_{1} t+Y_{1}(t) \sin \omega_{1} t, \text { is } \\
f_{U}(u)=\frac{1}{\sqrt{2 \pi} \sigma_{U}} \exp \left[-\frac{1}{2}\left(\frac{u-\mu_{U}}{\sigma_{U}}\right)^{2}\right] .
\end{gathered}
$$

For the random process

$$
P(t)=\sqrt{X_{2}^{2}(t)+Y_{2}^{2}(t)},
$$

we obtain ${ }^{1}$

$f_{P}(\rho)$

$$
\begin{aligned}
= & \frac{\rho}{2 \pi \sigma_{X_{2}} \sigma_{Y_{2}}} \cdot \exp \left[-\frac{1}{2}\left(\frac{\rho^{2}+2 \mu_{X_{2}}^{2}}{2 \sigma_{X_{2}}^{2}}+\frac{\rho^{2}+2 \mu_{Y_{2}}^{2}}{2 \sigma_{Y_{2}}^{2}}\right)\right] \\
& \times \int_{0}^{2 \pi} \exp \left[-\frac{1}{2}(\alpha \cos 2 \phi+c \cos \phi+d \sin \phi)\right] d \phi,
\end{aligned}
$$

where

$$
\begin{aligned}
& a=\rho^{2}\left[\left(2 \sigma_{X_{2}}^{2}\right)^{-1}\left(2 \sigma_{Y_{2}}^{2}\right)^{-1}\right], \\
& b=-\rho^{2}\left(\rho_{X Y} / \sigma_{X_{2}} \sigma_{Y_{2}}\right)=0 \\
& c=-2 \rho\left(\mu_{X_{2}} / \sigma_{X_{2}}^{2}\right)
\end{aligned}
$$

and

$$
d=-2 \rho\left(\mu_{Y_{2}} / \sigma_{Y_{2}}^{2}\right),
$$

and $\mu_{X_{2}}, \mu_{Y_{2}}$ are the expected values of $X_{2}(t)$ and $Y_{2}(t)$, respectively. A single convolution integral then yields the first-order PDF of $S(t)$ :

$$
f_{S}(s)=\int_{0}^{\infty} f_{P}(\rho) \cdot f_{U}(s-\rho) d \rho .
$$

\section{CALCULATION OF THE JOINT PDF}

The independent processes $X_{1}(t)$ and $Y_{1}(t)$ are Gaussian and stationary with known means and autocorrelations. Then

$$
U \equiv U(t)=X_{1}(t) \cos \omega_{1} t+Y_{1}(t) \sin \omega_{1} t
$$
and

$$
\begin{aligned}
U^{\prime} \equiv U(t+\tau)= & X_{1}(t+\tau) \cos \omega_{1}(t+\tau) \\
& +Y_{1}(t+\tau) \sin \omega_{1}(t+\tau)
\end{aligned}
$$

are also Gaussian random processes. Their means are, respectively,

$$
\mu_{U}=\mu_{X_{1}} \cos \omega_{1} t+\mu_{Y_{1}} \sin \omega_{1} t
$$

and

$$
\mu_{U^{\prime}}=\mu_{X_{1}} \cos \omega_{1}(t+\tau)+\mu_{Y_{1}} \sin \omega_{1}(t+\tau),
$$

while their second central moments are

$$
\sigma_{U}^{2}=\sigma_{X_{1}}^{2} \cos ^{2} \omega_{1} t+\sigma_{Y_{1}}^{2} \sin ^{2} \omega_{1} t
$$

and

$\sigma_{U^{\prime}}^{2}=\sigma_{X_{1}}^{2} \cos ^{2} \omega_{1}(t+\tau)+\sigma_{Y_{1}}^{2} \sin ^{2} \omega_{1}(t+\tau)$.

The correlation coefficient between $U$ and $U^{\prime}$ is

$$
\rho_{U}=\operatorname{Cov}\left(U, U^{\prime}\right) / \sigma_{U} \sigma_{U^{\prime}},
$$

where 


$$
\begin{aligned}
C_{U}(\tau) & \equiv \operatorname{Cov}\left(U, U^{\prime}\right) \\
& =E[U(t+\tau) U(t)]-E[U(t+\tau)] E[U(t)]
\end{aligned}
$$

or

with $^{12}$

$$
\begin{aligned}
C_{U}(\tau)= & C_{X_{1}}(\tau) \cos \omega_{1}(t+\tau) \cos \omega_{1} t \\
& +C_{Y_{1}}(\tau) \sin \omega_{1}(t+\tau) \sin \omega_{1} t
\end{aligned}
$$

$$
\begin{aligned}
& C_{X_{1}}(\tau)=E\left\{\left[X_{1}(t+\tau)-\mu_{X_{1}}\right]\left[X_{1}(t)-\mu_{X_{1}}\right]\right\} \\
& C_{Y_{1}}(\tau)=E\left\{\left[Y_{1}(t+\tau)-\mu_{Y_{1}}\right]\left[Y_{1}(t)-\mu_{Y_{1}}\right]\right\} .
\end{aligned}
$$

Then their joint PDF is

$$
f_{U U^{\cdot}}\left(u, u^{\prime}\right)=\frac{1}{2 \pi \sigma_{U} \sigma_{U}^{\prime}\left(1-\rho_{U}^{2}\right)^{1 / 2}} \cdot \exp \left[-\frac{1}{1-\rho_{U}^{2}}\right.
$$

$$
\begin{aligned}
& \times\left(\frac{\left(U-\mu_{U}\right)^{2}}{2 \sigma_{U}^{2}}-\frac{\rho_{U}\left(u-\mu_{U}\right)\left(u^{\prime}-\mu_{U^{\prime}}\right)}{\sigma_{U} \sigma_{U^{\prime}}}\right. \\
& \left.\left.+\frac{\left(u^{\prime}-\mu_{U^{\prime}}\right)^{2}}{2 \sigma_{U^{\prime}}^{2}}\right)\right]
\end{aligned}
$$

Consider now $X_{2}(t)$ and $Y_{2}(t)$. They are also Gaussian, stationary, independent random processes with known means, autocorrelations, and cross correlation. Then the joint PDF of the random processes

$$
\begin{aligned}
& P=P(t)=\sqrt{X_{2}^{2}(t)+Y_{2}^{2}(t)}, \\
& P^{\prime}=P(t+\tau)=\sqrt{X_{2}^{2}(t+\tau)+Y_{2}^{2}(t+\tau)},
\end{aligned}
$$

is given $b^{2}$ :

$$
\begin{aligned}
f_{\rho \rho^{\prime}}\left(\rho, \rho^{\prime}\right)= & \frac{\rho \rho^{\prime}}{4 \pi^{2}\left|\Lambda_{2}\right|^{1 / 2}} \int_{0}^{2 \pi} \int_{0}^{2 \pi} \exp \left[-\frac{1}{2}\left(A+B \cos \theta_{1}+C \sin \theta_{1}+D \cos 2 \theta_{1}+E \sin 2 \theta_{1}+F \cos \theta_{2}+G \sin \theta_{2}\right.\right. \\
& \left.\left.+H \cos 2 \theta_{2}+I \sin 2 \theta_{2}+J \cos \theta_{1} \cos \theta_{2}+K \sin \theta_{1} \cos \theta_{2}+L \sin \theta_{1} \sin \theta_{2}+M \cos \theta_{1} \sin \theta_{2}\right)\right] d \theta_{1} d \theta_{2},
\end{aligned}
$$

where

$$
\begin{aligned}
A= & \lambda_{1} \rho^{2} / 2+\mu_{X_{2}} \lambda_{11}+2 \mu_{X_{2}} \mu_{Y_{2}} \lambda_{12}+2 \lambda_{13} \mu_{X_{2}}^{2} \\
& +2 \lambda_{14} \mu_{X_{2}} \mu_{Y_{2}}+\lambda_{22} \rho^{2} / 2+\mu_{Y_{2}}^{2} \lambda_{22} \\
& +\lambda_{33} \rho^{\prime 2}+\mu_{X_{2}} \lambda_{33}+2 \mu_{X_{2} Y_{2}} \lambda_{34}+2 \lambda_{24} \mu_{Y_{2}}^{2} \\
& +2 \lambda_{23} \mu_{X_{2} Y_{2}}+\lambda_{4 \rho^{2}} \rho^{2} / 2+\mu_{Y_{2}}^{2} \lambda_{44} \\
B= & -2 \lambda_{1} \rho \mu_{X_{2}}-2 \lambda_{12} \rho \mu_{Y_{1}}-2 \lambda_{13} \rho \mu_{X_{2}}-2 \lambda_{14} \rho \mu_{Y_{2}}, \\
C= & -2 \lambda_{12} \rho \mu_{X_{2}}-2 \lambda_{22} \rho \mu_{Y_{2}}-2 \lambda_{23} \rho \mu_{X_{2}}-2 \lambda_{24} \rho \mu_{Y_{2}}, \\
D= & \lambda_{1} \rho^{2} / 2-\lambda_{22} \rho^{2} / 2, \\
E= & \lambda_{12} \rho^{2}, \\
F= & -2 \lambda_{13} \rho^{\prime} \mu_{X_{2}}-2 \lambda_{23} \rho^{\prime} \mu_{Y_{2}} \\
& -2 \lambda_{33} \rho^{\prime} \mu_{X_{2}}-2 \lambda_{34} \rho^{\prime} \mu_{Y_{2}}, \\
G= & -2 \lambda_{14} \rho^{\prime} \mu_{X_{2}}-2 \lambda_{24} \rho^{\prime} \mu_{Y_{2}} \\
& -2 \lambda_{34} \rho^{\prime} \mu_{X_{2}}-2 \lambda_{44} \rho^{\prime} \mu_{Y_{2}}, \\
H= & \lambda_{33} \rho^{\prime 2} / 2-\lambda_{44} \rho^{\prime 2} / 2, \\
I= & \lambda_{34} \rho^{\prime 2}, \\
J= & 2 \lambda_{13} \rho \rho^{\prime}, \\
K= & 2 \lambda_{23} \rho \rho^{\prime}, \\
L= & 2 \lambda_{24} \rho \rho^{\prime}, \\
M= & 2 \lambda_{14} \rho \rho^{\prime},
\end{aligned}
$$

with $\lambda_{i j}$ being elements of the inverse covariance matrix $\left[\Lambda_{2}\right]^{-i}$. The covariance matrix itself is given by

$$
\left[\Lambda_{2}\right]=\left[\begin{array}{cccc}
C_{X_{2}}(0) & C_{X_{2} Y_{2}}(0) & C_{X_{2}}(\tau) & C_{Y_{2} X_{2}}(\tau) \\
C_{Y_{2} X_{2}}(0) & C_{Y_{2}}(0) & C_{X_{1} Y_{2}}(\tau) & C_{Y_{1}}(\tau) \\
C_{X_{1}}(\tau) & C_{X_{2} Y_{2}}(\tau) & C_{X_{2}}(0) & C_{X_{2} Y_{2}}(0) \\
C_{Y_{2} X_{2}}(\tau) & C_{Y_{2}}(\tau) & C_{X_{2} Y_{2}}(0) & C_{Y_{Y}}(0)
\end{array}\right],
$$

where

$$
C_{X_{2}}(\tau)=E\left\{\left[X_{2}(t+\tau)-\mu_{X_{2}}\right]\left[X_{2}(t)-\mu_{X_{2}}\right]\right\},
$$

$$
\begin{aligned}
& C_{Y_{2}}(\tau)=E\left\{\left[Y_{2}(t+\tau)-\mu_{Y_{2}}\right]\left[Y_{2}(t)-\mu_{Y_{2}}\right]\right\}, \\
& C_{X_{2} Y_{2}}(\tau)=E\left\{\left[X_{2}(t+\tau)-\mu_{X_{2}}\right]\left[Y_{2}(t)-\mu_{Y_{2}}\right]\right\}, \\
& C_{Y_{2} X_{2}}(\tau)=E\left\{\left[Y_{2}(t+\tau)-\mu_{Y_{2}}\right]\left[X_{2}(t)-\mu_{X_{2}}\right]\right\} .
\end{aligned}
$$

Finally, if we define

$$
S \equiv S(t) \text { and } S^{\prime} \equiv S(t+\tau),
$$

a double convolution integral will yield the desired result:

$f_{S s^{\prime}}\left(s, s^{\prime}\right)=\int_{0}^{\infty} \int_{0}^{\infty} f_{U U^{\prime}}\left(s-\rho, s^{\prime}-\rho^{\prime}\right) \cdot f_{\rho \rho^{\prime}}\left(\rho, \rho^{\prime}\right) d \rho d \rho^{\prime}$.

Let us now focus on the maxima of our process $Z(t)$, in the time interval $T$, which cannot be analytically located. Consequently, the number of maxima of our process $Z(t)$, denoted by $v$, cannot be calculated analytically. Hence, we are going to adopt an approximate approach. We know that

$$
Z(t)=W_{1}(t)+W_{2}(t)
$$

Since they are narrow band, the time interval between two successive local maxima is approximately constant for each process. Therefore, for process $W_{1}(t)$, the expected period between successive maxima is

$$
\tau_{1}=2 \pi / \omega_{1},
$$

and the expected number of maxima in the time interval $T$ is one plus the number of cycles in the interval, that is, ${ }^{5}$

$$
v_{1}=T / \tau_{1}+1
$$

Similarly, for the process $W_{2}(t)$, the expected period between successive maxima is

$$
\tau_{2}=2 \pi / \omega_{2},
$$

and the expected number of maxima is

$$
v_{2}=T / \tau_{2}+1 \text {. }
$$

If we now make the assumption that $\omega_{1}$ is sufficiently small, $\tau_{1}$ becomes very large and does not affect the number of 
maxima. In this case, we can approximate the number of maxima of $Z(t)$ as

$$
v=v_{2}=T / \tau_{2}+1 \text {. }
$$

\section{NUMERICAL EXAMPLE}

In order to verify the above theoretical results we tried several numerical examples using the codes developed, one of which is discussed below.

The data that we used are the following (we chose the data in such a way, that the final probability is a finite number):

$$
\begin{aligned}
& \text { upcrossing level }=5.00000 \text {, } \\
& \omega_{1}=3.14159 \text {, } \\
& t=1.00000, \quad \tau=1.40000 \\
& \mu_{X_{1}}=-0.00300, \quad \mu_{Y_{1}}=0.01600 \text {, } \\
& \mu_{X_{2}}=-0.00300, \quad \mu_{Y_{2}}=0.01600 \text {, } \\
& \sigma_{X_{1}}=2.36800, \quad \sigma_{Y_{1}}=2.36800 \text {, } \\
& C_{X_{1}}(\tau)=1.94652, \quad C_{Y_{1}}(\tau)=1.94652 \text {, } \\
& {\left[\Lambda_{X_{2} Y_{2}}\right]} \\
& =\left[\begin{array}{llll}
2.36800 & 0.00000 & 1.87800 & 0.00000 \\
0.00000 & 2.36800 & 0.00000 & 1.87800 \\
1.87800 & 0.00000 & 2.36800 & 0.00000 \\
0.00000 & 1.87800 & 0.00000 & 2.36800
\end{array}\right] .
\end{aligned}
$$

Ref. 5.

We also used the value of 1.2 for variable $v$, taken from

We ran the program several times using different values for the number of points of integration. The fact that three nested numerical integrations are involved in the evaluation of the coefficient $c$ and six are involved in the evaluation of $b$ makes the overall computations quite time consuming.

The variable $N T 1$ represents the number of points used in the double convolution integral (24).

The variables $N T 2$ and $N T 3$ are the equivalent numbers for the integration over $\theta_{1}$ and $\theta_{2}$, respectively. In this examplewe always had: $N T 2=N T 3$. Here, $N T 4$ is the number of points used in the double integration of (15). We set $N T 1=N T 4$. Finally, $N T 5$ is the number of points used in all the integrations involved in calculation of the coefficient $c$.

Table I gives the value of the coefficient $b$ and the up-

TABLE I. The value of the coefficient $b$ and the upcrossing probability $p$ for different combinations of $N T 1, N T 2, N T 3$, and $N T 4$, with $N T 5=50$.

\begin{tabular}{ccccc}
\hline \hline$N T 2 / N T 3 / N T 1 / N T 4$ & 5 & 10 & 20 \\
\hline 5 & $b$ & 0.29132 & 0.21138 & 0.21568 \\
& $p$ & 0.28341 & 0.26793 & 0.26872 \\
10 & $b$ & 0.16023 & 0.13519 & 0.13954 \\
& $p$ & 0.25867 & 0.25430 & 0.25505 \\
20 & $b$ & 0.15045 & 0.12811 & 0.13247 \\
& $p$ & 0.25695 & 0.25308 & 0.25383 \\
\hline \hline
\end{tabular}

TABLE II. The value of the coefficient $C$ and the upcrossing probability $p$ for $N T 1=N T 2=N T 3=N T 4=5$ points, with $N T 5$ ranging from 10 to 50 points.

\begin{tabular}{cccccc}
\hline \hline NT 5: & 10 & 20 & 30 & 40 & 50 \\
\hline$c:$ & 0.23211 & 0.23229 & 0.23231 & 0.23232 & 0.23232 \\
$p:$ & 0.28320 & 0.28338 & 0.28340 & 0.28341 & 0.28341 \\
\hline \hline
\end{tabular}

crossing probability $p$ for different combinations of $N T 1$, $N T 2, N T 3$, and $N T 4$, with $N T 5=50$.

The main problem is computational effort: For $5 \times 5$ points this was not excessive, but as the number of points increased (together with accuracy) the time required increased to $40 \mathrm{~min}$ for $10 \times 10$ points, to $18 \mathrm{~h}$ of computer time for the $20 \times 20$ case. (These refer to an APOLLO-DOMAIN Series 3000 -computer, but could be used in a comparative sense even if a different computer is employed.)

Nevertheless, we can easily observe from Table I that accuracy increases fairly fast, since $b$ tends to stabilize at about 0.13 , with probability $p$ at 0.25 approximately, as soon as $10 \times 10$ points of integration are used. Furthermore, we can see that the value of $p$ is not as dramatically influenced by the numbers NT1-NT4, as that of $b$. This can be explained if we recall the formula we derived for $p,(13)$. Yet the value of $p$ is influenced by $N T 5$, as one can see either from (13) or from Table II. To form this table, we set $N T 1=N T 2=N T 3=N T 4=5$ points, with $N T 5$ ranging from 10 to 50 points. It is easily observed that we get a steady-state value for $c$ from 40 points on and for $p$ from as low as 40 points, too.

Closing this brief discussion, we can conclude that 40 (or 50) points for any single integration would give very good results, but even 20 points should provide adequate accuracy.

\section{CONCLUSIONS}

We developed an approach for the evaluation of the level crossing probability of a random process of the form (1). Processes $X_{i}(t)$ and $Y_{i}(t)$ are jointly Gaussian, jointly stationary, and jointly independent, with known means and autocorrelations. The above probability has been calculated for some known time interval $T$. Our approach was based on the approximation of the local maxima of $Z(t)$ by the value of the associated envelope. The times that the maxima occur define a two-state discrete-time process defined by the requirements that the value of $Z(t)$ exceeds or does not exceed the threshold $Z_{0}$. This process can be assumed to be Markov with one step memory. Independence of maxima can also be assumed for this process, in order to facilitate the calculations. The level crossing probability was finally given.

\section{ACKNOWLEDGMENTS}

The authors would like to thank the Michigan and National Sea Grant College Programs for the funding of an ongoing research project, the first stages of which are reported in this paper. The inputs of Professor M. G. Parsons of the University of Michigan and Professor E. Nikolaidis of Vir- 
ginia Polytechnic Institute and State University are also appreciated.

'P. N. Mikhalevsky, "Envelope Statistics of Partially Saturated Processes," J. Acoust. Soc. Am. 72, 151-158 (1982).

${ }^{2}$ E. Nikolaidis and A. N. Perakis, "Second-Order Statistics and DiscreteTime Detection Modeling for Partially Saturated Processes," J. Acoust. Soc. Am. 77, 1078-1085 (1985).

${ }^{3}$ H. N. Psaraftis, A. N. Perakis, and N. Mikhalevsky, "New Models on the Ocean Acoustic Detection Process," J. Acoust. Soc. Am. 69, 1724-1734 (1981).

A. N. Perakis and H. N. Psaraftis, "Diserete-Time Modeling of the Unsaturated Detection Process," J. Acoust. Soc. Am. 74, 1630-1633 (1983). ${ }^{5}$ E. Nikolaidis, A. N. Perakis, and M.G. Parsons, "Structural Reliability of Marine Diesel Engine Propulsion Shafting Systems," presented at the November 1985 SNAME Annual Meeting and included in the Trans. Soc. Naval Architects Marine Engineers 93, 189-223 (1985).
${ }^{6}$ E. Nikolaidis, A. N. Perakis, and M. G. Parsons, “Probabilistic Torsional Vibration Analysis of a Marine Diesel Engine Shafting System: The Level Crossing Problem," submitted for publication to the J. Appl. Mech. 56, 953-959 (1989).

'E. Nikolaidis, A. N. Perakis, and M. G. Parsons, "Structural Reliability of Diesel Engine Shafting Systems," in Advanced Diesel Engineering, edited by S. Haddad (Wiley, New York, 1987).

${ }^{\text {g}}$ H. Cramer and M. R. Leadbetter, Stationary and Related Stochastic Processes (Wiley, New York, 1967).

${ }^{9} \mathrm{G}$. R. Toro and C. A. Cornell, "Extremes of Gaussian Processes with Bimodal Spectra," J. Eng. Mech. 112, 465-484 (1986).

${ }^{10}$ L. D. Lutes and S.-H. Tzuang, "First-Passage Probability for Two-Mode Systems," J. Eng. Mech. 109, 1358-1374 (1983).

"B. Epstein, "The Distribution of Extreme Values in Sample Whose Members are Subject to the Markov Chain Condition," Ann. Math. Stat. 20, 59-594 (1949).

${ }^{12}$ A. Papoulis, Probability, Random Variables and Stochastic Processes (McGraw-Hill, New York, 1965). 